\title{
Title
}

Higher liver stiffness is associated with early kidney dysfunction in patients with

histologically-proven non-cirrhotic NAFLD

Short title: Liver stiffness and early kidney disease

\section{Authors' name}

Dan-Qin Sun ${ }^{1,2 \#}$, Fang-Zhou Ye ${ }^{3,4 \#}$, Haluk Tarik Kani ${ }^{5 \#}$, Jian-Rong Yang ${ }^{6}$, Kenneth I. Zheng ${ }^{3}$, HaoYang Zhang ${ }^{7}$, Giovanni Targher ${ }^{8}$, Christopher D. Byrne', Yong-Ping Chen ${ }^{3,10}$, Wei-Jie Yuan ${ }^{2}$, Yusuf Yilmaz ${ }^{5,11^{*}}$ and Ming-Hua Zheng ${ }^{3,10^{*}}$

\section{Institution:}

${ }^{1}$ Department of Nephrology, the Affiliated Wuxi No.2 People's Hospital of Nanjing Medical University, Wuxi, China

${ }^{2}$ Department of Nephrology, Shanghai General Hospital of Nanjing Medical University, Shanghai, China

${ }^{3}$ NAFLD Research Center, Department of Hepatology, the First Affiliated Hospital of Wenzhou Medical University, Wenzhou, China

${ }^{4}$ School of the First Clinical Medical Sciences, Wenzhou Medical University, Wenzhou, China ${ }^{5}$ Department of Gastroenterology, Marmara University School of Medicine, Istanbul, Turkey ${ }^{6}$ Department of Clinical Laboratory, the First Affiliated Hospital of Wenzhou Medical University, Wenzhou, China

${ }^{7}$ School of Biomedical Engineering, Sun Yat-sen University, Guangzhou, China

${ }^{8}$ Division of Endocrinology, Diabetes and Metabolism, Department of Medicine, University and Azienda Ospedaliera Universitaria Integrata of Verona, Verona, Italy

${ }^{9}$ Southampton National Institute for Health Research Biomedical Research Centre, University Hospital Southampton, Southampton General Hospital, Southampton, UK

${ }^{10}$ Institute of Hepatology, Wenzhou Medical University, Wenzhou, China

${ }^{11}$ Institute of Gastroenterology, Marmara University, Istanbul, Turkey 
Co-first author: Dan-Qin Sun, Fang-Zhou Ye, Haluk Tarik Kani

\section{*Co-corresponding author:}

Ming-Hua Zheng, MD, PhD

NAFLD Research Center, Department of Hepatology, the First Affiliated Hospital of Wenzhou Medical University; No. 2 Fuxue Lane, Wenzhou 325000, China.

E-mail: zhengmh@wmu.edu.cn; fax: (86) 577-55579622; tel: (86) 577-55578522.

Yusuf Yilmaz, MD

Department of Gastroenterology, Marmara University School of Medicine, Istanbul 34662, Turkey E-mail: yusufyilmaz@uludag.edu.tr

Guarantor of the article: Ming-Hua Zheng

\section{List of Abbreviations}

$\mathrm{ACR}=$ albumin-to-creatinine ratio, $\mathrm{AUROC}=$ area under the receiver operating characteristic curves, $\mathrm{BMI}=$ body mass index, $\mathrm{CI}=$ confidence interval, $\mathrm{CKD}=$ chronic kidney disease, $\mathrm{eGFR}=$ estimated glomerular filtration rate, EKD = early kidney dysfunction, HOMA-IR = homeostasis model assessment-insulin resistance, $\mathrm{NAFLD}=$ non-alcoholic fatty liver disease, $\mathrm{OR}=$ odds ratio, $\mathrm{LSM}=$ liver stiffness measurement, $\mathrm{LF}=$ liver fibrosis, $\mathrm{TE}=$ transient elastography 


\begin{abstract}
Aim: We examined the association between liver fibrosis (LF), assessed either by histology or by liver stiffness measurement (LSM), and the presence of early kidney dysfunction (EKD). We also investigated the diagnostic performance of LSM for identifying the presence of EKD in patients with nonalcoholic fatty liver disease (NAFLD).
\end{abstract}

Materials and Methods: We studied 214 adults with non-cirrhotic biopsy-proven NAFLD recruited from two independent medical centers. Histologic stage of LF was quantified using the Brunt's criteria. Vibration-controlled transient elastography (TE) was performed in 154 patients and defined as significant when LSM was $\geq 8.0 \mathrm{kPa}$ using the M probe (Fibroscan ${ }^{\circledR}$ ). EKD was defined by the presence of microalbuminuria with an estimated glomerular filtration rate $\geq 60 \mathrm{ml} / \mathrm{min} / 1.73 \mathrm{~m}^{2}$. Logistic regression modelling was undertaken to estimate the likelihood of having EKD with NAFLD (the LSM-EKD model).

Results: The prevalence of EKD was higher in patients with LF than in those without LF on histology (22.14\% vs. 4.82\%, $P<0.001)$. Similarly, the prevalence of EKD was higher in patients with $\mathrm{LSM} \geq 8.0 \mathrm{kPa}$ than in those with $\mathrm{LSM}<8.0 \mathrm{kPa}(23.81 \%$ vs. $6.59 \%, P<0.05)$, and the area under the ROC curve of the LSM-EKD model for identifying EKD was 0.80 (95\%CI 0.72-0.89). LF, detected by either method, was associated with EKD independently of established renal risk factors and potential confounders.

Conclusions: LF is independently associated with EKD in patients with biopsy-proven NAFLD. TE-measured LSM is a widely used technique for quantifying LF, which accurately identifies those patients with NAFLD, who are at risk of having EKD.

Key-words: Non-alcoholic fatty liver disease, early kidney dysfunction, liver fibrosis, liver 
stiffness measurement 


\section{Introduction}

Nonalcoholic fatty liver disease (NAFLD) is a major cause of liver-related mortality and increases the risk of developing chronic kidney disease (CKD) [1-3]. CKD is a general term for heterogeneous diseases affecting both the kidney structure and function and CKD is also often associated with an increased risk of cardiovascular events and all-cause mortality [4]. Progression to end-stage kidney disease can only be treated with chronic dialysis or renal transplantation, causing a considerable economic burden on health services [5]. The early stages of CKD are often asymptomatic and may be detected incidentally during the evaluation of other diseases [6]. As the early stages of CKD may be reversible, their early detection is clinically important.

In recent years, several studies have examined the association between NAFLD and the risk of CKD supporting the conclusion that the presence and severity of NAFLD, diagnosed either by imaging or histology, is associated with an increased prevalence and incidence of CKD $[1,7,8]$. Although these studies have provided evidence for a strong association between the presence and severity of NAFLD and risk of stage 3-5 CKD [7-9], there are currently very few studies that have examined the association between NAFLD and the early stages of kidney dysfunction (EKD, defined as presence of microalbuminuria with an estimated glomerular filtration $[\mathrm{eGFR}] \geq 60$ $\left.\mathrm{ml} / \mathrm{min} / 1.73 \mathrm{~m}^{2}\right)$.

Transient elastography (TE) has been extensively used as a non-invasive technique for measuring liver fibrosis (LF) and LF may identify a subgroup of patients with NAFLD who are at higher risk of liver-related clinical outcomes, including requirement for liver transplantation [10-12]. The diagnostic value of liver stiffness measurement (LSM), obtained from TE, in identifying EKD among patients with NAFLD is currently uncertain. Therefore, the major aim of our study was to examine the association between the severity of LF (assessed invasively by liver histology; or noninvasively by TE) and the presence of EKD in patients with biopsy-proven NAFLD. Additionally, 
we have also investigated the diagnostic performance of both LF assessed by histology and LSM assessed by TE for predicting the presence of EKD in this patient population.

\section{Materials and Methods}

\section{Study design}

Patients who had undergone liver biopsy were from two medical centers: First Affiliated Hospital of Wenzhou Medical University, China and Marmara University School of Medicine, Turkey. Of these 742 adult patients who underwent liver biopsy, 214 patients with biopsy-confirmed NAFLD were included in the study. As shown in Figure 1, in both centers we excluded patients who had any of following criteria: excessive alcohol consumption ( $>140 \mathrm{~g} /$ week for men and $>70 \mathrm{~g} / \mathrm{week}$ for women), urinary tract infections, were febrile or were menstruating. Participants with other known causes of chronic liver disease (such as viral hepatitis, autoimmune hepatitis, primary biliary cholangitis or Wilson's disease - these conditions were excluded on the basis of liver biopsy results as well as by testing for serological markers of hepatitis A, B and C, anti-mitochondrial antibodies, smooth muscle cell antibodies, anti-nuclear antibodies, anti-liver kidney microsomal antibodies and levels of caeruloplasmin), prior history of cirrhosis of any etiology, CKD stage 3-5, macroalbuminuria (i.e., urinary albumin-to-creatinine ratio $>300 \mathrm{mg} / \mathrm{g}$ ) or other known causes of kidney disease were also excluded from the analysis.

Written informed consent was obtained from each subject before their participation in the study. Personal information was removed and all study participants were identified only by their health examination number. The research protocol in Wenzhou was approved by the ethics committee of the First Affiliated Hospital of Wenzhou Medical University. The local Institutional Review Board approved the study in Turkey.

\section{Liver biopsy and transient elastography}


Ultrasound-guided liver biopsy was performed under sedation using a 16-gauge Hepafix needle (Gallini, Modena, Italy). All biopsy specimens were placed in formalin solution for fixation, embedded in paraffin blocks and stained with hematoxylin eosin and Masson's trichrome. All liver pathology slides from Wenzhou and Istanbul were assessed by a single experienced liver pathologist who was blinded to laboratory and clinical data of participants. The histologic stage of LF was quantified using the Brunt's criteria [13] as follows: stage 1, zone 3 peri-sinusoidal fibrosis; stage 2, as for stage 1 with portal fibrosis; stage 3, as for stage 2 with bridging fibrosis; and stage 4 , cirrhosis. By study design, patients with cirrhosis were excluded from the study because these patients have a low skeletal muscle mass and a lower serum creatinine level making the accurate estimation of eGFR more difficult in this patient group. Patients were then stratified into two groups according to their histologic stage of LF: no-LF (stage F=0) and LF (stages F 1 to 3).

Overall, 154 of $214(72 \%)$ patients were also assessed by vibration-controlled transient elastography (TE) (Fibroscan ${ }^{\circledR}$, Echosens, Paris, France). This technique utilizes a probe, which generates a pressure wave and an ultrasound wave. The propagation of the pressure wave depends on the stiffness of the liver tissue while the propagation of the ultrasound wave is largely affected by the amount of adipose tissue. Patients were predicted to have significant LF if they had a LSM $\geq 8.0$ $\mathrm{kPa}$ as measured by the $\mathrm{M}$ probe, which has been shown to be equivalent to the Kleiner $\mathrm{F} \geq 2$ stage on liver histology $[10,14]$.

\section{Clinical and laboratory data}

Anthropometric and clinical parameters and comorbidities were recorded from all participants. Body mass index (BMI) was calculated as weight in kilograms divided by height in meters squared. Blood pressure was measured using an automated sphygmomanometer with the subject in a quiet environment and in a sitting position for at least $10 \mathrm{~min}$. Hypertension was defined as blood pressure $\geq 130 / 85 \mathrm{mmHg}$ or the use of any anti-hypertensive drugs. Diabetes was defined by a 
fasting plasma glucose level $\geq 7.0 \mathrm{mmol} / \mathrm{L}$ or $\mathrm{HbA} 1 \mathrm{c} \geq 6.5 \%$, or the use of antihyperglycemic agents. Hyperuricemia was defined as a serum uric acid level $>420 \mu \mathrm{mol} / \mathrm{L}$ for men and $>360$ $\mu \mathrm{mol} / \mathrm{L}$ for women, respectively, or use of allopurinol. Dyslipidemia was defined as any of the following criteria: total cholesterol $>5.17 \mathrm{mmol} / \mathrm{L}$; triglycerides $>1.70 \mathrm{mmol} / \mathrm{L}$; high-density lipoprotein cholesterol (HDL-c) $<1.0 \mathrm{mmol} / \mathrm{L}$ for women and $<1.3 \mathrm{mmol} / \mathrm{L}$ for men and lowdensity lipoprotein cholesterol (LDL-c) $\geq 3.4 \mathrm{mmol} / \mathrm{L}$, or the use of any lipid-lowering drugs. Homeostasis model assessment of insulin resistance (HOMA-IR score) was calculated using fasting glucose and insulin measurements as follows: [fasting insulin $(\mathrm{mU} / \mathrm{ml}) \times$ fasting glucose $(\mathrm{mmol} / \mathrm{L}) / 22.5]$. Biochemical laboratory parameters included measurement of albumin, alanine aminotransaminase (ALT), aspartate aminotransferase (AST), glucose, blood urea nitrogen, creatinine, uric acid, lipids, white blood cell count, red blood cell count and platelet count. All these parameters were measured by an automated analyzer (Abbott AxSYM), using standard laboratory methods. EKD was defined by the presence of microalbuminuria (i.e., urinary albumin-to-creatinine ratio $30-300 \mathrm{mg} / \mathrm{g}$ ) with an eGFR value $\geq 60 \mathrm{ml} / \mathrm{min} / 1.73 \mathrm{~m}^{2}$ as estimated using the CKD-EPI equation [15].

\section{Statistical analysis}

Data are presented as means \pm standard deviation (SD) or frequencies. Baseline clinical and biochemical characteristics of the study population were compared using the one-way analysis of variance (ANOVA) for continuous variables and the $\chi^{2}$-test for categorical variables. The association between LF, assessed either by histology or by TE, and the presence of EKD in patients with NAFLD, was tested using both an unadjusted logistic regression model and the following two multivariable logistic regression models with pre-specified adjustments: (a) model adjusted for age, sex, ethnicity, waist circumference and hip circumference; and (b) model further adjusted for hyperuricemia, dyslipidemia, hypertension, diabetes and HOMA-IR score. 
Additionally, to identify possible predictors of EKD, two logistic regression models: a) LF identified by the histology model (named the LFH-EKD model), and b) LF identified by the TE model (named the LSM-EKD model) were constructed to generate the probability of having EKD for each patient with NAFLD. The two models used the following equation probability $=\exp (\mathrm{c}) /$ $(1+\exp (\mathrm{c}))$, where $\mathrm{c}$ is $1.63 \times \mathrm{LF}-\mathrm{histology}($ stage $\mathrm{F} 1-3$ is 1 or stage $\mathrm{F}=0$ is 0$)-0.84 \times \operatorname{sex}($ male $=1$ or female $=0)+0.02 \times$ age -3.45 in the LFH-EKD model, or $\mathrm{c}$ is $1.43 \times$ LF-TE $(\mathrm{LSM} \geq 8 \mathrm{kPa}$ is 1 or $\mathrm{LSM}<8 \mathrm{kPa}$ is 0$)-1.70 \times \mathrm{sex}($ male $=1$ or female $=0)+0.01 \times$ age -2.207 in the LSM-EKD model. Moreover, we examined the discrimination in the context of logistic regression analyses using the area under the receiver operating characteristic curve (AUROC) by MedCalc version 12.7 (MedCalc Software, Ostend, Belgium). The optimal cut-off point was identified based on the maximal Youden index (sensitivity + specificity -1$)$. All statistical tests were two-sided and $P$ value of $<0.05$ (two-tailed) was considered statistically significant. The statistical analyses were conducted using the SPSS version 22.0 (SPSS, Chicago, IL).

\section{Results}

Among the 214 patients with biopsy-proven NAFLD included in the study, the prevalence of LF and EKD were $15.4 \%$ and $61.2 \%$, respectively. Patients in the LF group (assessed by liver biopsy) had higher adiposity measures (BMI, waist circumference and hip circumference) and lower eGFR levels than those in the non-LF group (all $P<0.05$ ). In contrast, no significant differences were found in serum uric acid levels, plasma lipid profile and HOMA-IR score between the two groups of patients (Supplementary table 1).

Table 1 shows the main clinical and biochemical characteristics of NAFLD patients stratified by EKD status. Patients with EKD were more likely to be older, centrally obese and hypertensive, and had lower eGFR levels and a greater histologic stage of LF $(P<0.05)$ (Table 1). The mean values of systolic and diastolic blood pressure as well as HOMA-IR score were higher in subjects with EKD 
compared to those without EKD (all $P<0.05$ ). Subjects with EKD also had a greater prevalence of diabetes and hypertension than those without EKD.

To verify the relationship between LF and EKD, we have evaluated the prevalence of EKD in our NAFLD participants. The prevalence of EKD was higher in the LF group than that in the non-LF group (22.14\% vs. $4.82 \%, P<0.001)$. Notably, as shown in Table 2, the histology-measured LF was significantly associated with presence of EKD (adjusted-odds ratio 4.33, 95\%CI 1.25-15.03; $P=0.021$, even after adjustment for age, sex, ethnicity, waist circumference, hip circumference, hypertension, dyslipidemia, hyperuricemia, diabetes and HOMA-IR score.

Overall, $72 \%(n=154)$ of patients also underwent a TE examination. The prevalence of TEmeasured significant LF (i.e., defined as LSM $\geq 8.0 \mathrm{kPa}$ and no less than stage F2 on histology) was lower than that measured by liver biopsy (40.90\% vs. $61.21 \%)$. However, the TE-measured LF showed good diagnostic performance for predicting the histologic stage of LF (stage F 1-3) (AUROC $=0.75,95 \%$ CI 0.68-0.82) (Figure 2A). The association between TE-measured LF and EKD was consistent with that observed with liver biopsy results. Notably, compared to those with LSM $<8.0 \mathrm{kPa}$, patients with LSM $\geq 8.0 \mathrm{kPa}$ had a higher prevalence of EKD $(23.81 \% \mathrm{vs} .6 .59 \%$, $P<0.05$ ) (Table 3).

As shown in Table 2, similar to the association between the histology-measured LF and EKD, the association between TE-measured LF and risk of EKD remained statistically significant (adjusted OR 4.73, 95\%CI 1.19-28.48, $P=0.03$ ) even after adjustment for age, sex, ethnicity, waist circumference, hip circumference, hypertension, dyslipidemia, hyperuricemia, diabetes and HOMA-IR score.

To further examine the diagnostic accuracy of: a) LF identified by histology and b) LF identified by 
TE for identifying EKD, we constructed two different models, i.e. the LFH-EKD model and the LSM-EKD model, as specified above in the Statistical analysis section, with three variables (sex, age and either LF stage or LSM score) that are widely available in clinical practice. Notably, as shown in Figure 2B and 2C, the LSM-EKD model accurately identified the presence of EKD (AUROC $0.80,95 \%$ CI $0.72-0.89, P<0.05$ ), and performed slightly better in identifying EKD compared to the similar LFH-EKD model. When we used an optimal cutoff point of 0.11 according to the best Youden index, the associated sensitivity and specificity for the LSM-EKD model were $90.5 \%$ and $62.4 \%$ respectively.

\section{Discussion}

The novel findings of our cross-sectional study are as follows: (a) the severity of LF, assessed by either histology or TE, was significantly associated with an increased risk of prevalent EKD (i.e. defined as presence of microalbuminuria with an eGFR value $\geq 60 \mathrm{ml} / \mathrm{min} / 1.73 \mathrm{~m}^{2}$ ) even after adjustment for age, sex, ethnicity, adiposity measures, hypertension and other established renal risk factors; (b) the significant association between TE-measured LF and risk of EKD was consistent with histological data obtained from liver biopsy; and (c) both the LFH-EKD model and the LSMEKD model showed good diagnostic performances for accurately identifying EKD. We believe that these results are clinically important because TE, i.e., a reliable, non-invasive method for staging LF, appears to be also useful for accurately identifying NAFLD patients, who are at increased risk of EKD.

Our findings expand the results of some cross-sectional Asian studies that have been recently published by other investigators. In a cohort of 1763 Chinese patients with type 2 diabetes from Hong Kong, Yeung et al. reported that advanced fibrosis, assessed by TE, was independently associated with an increased risk of albuminuria $\geq 30 \mathrm{mg} / \mathrm{g}$ (adjusted odds ratio $=1.52,95 \% \mathrm{CI}$ : 1.02 2.28, $P=0.039$ ) [14]. Similarly, another study including 1439 Chinese patients reported that 
increased LSM, assessed by TE, was a potential indicator for CKD (defined as eGFR $\leq 60$ $\mathrm{mL} / \mathrm{min} / 1.73 \mathrm{~m}^{2}$ ) in patients with ultrasound-detected NAFLD [12]. However, it should be noted that none of these two studies have used liver biopsy for diagnosing and staging of NAFLD and these two studies did not examine the association between the severity of LF and risk of EKD. Moreover, in our study, we found that the significant association between the severity of LF (assessed by either histology or TE) and risk of EKD persisted after adjustment for age, sex, adiposity measures, hypertension, HOMA-IR score and other established risk factors for CKD.

Many risk factors for NAFLD have the potential to influence the development of EKD. Firstly, the results of our study show that patients with NAFLD and EKD were more insulin resistant than those without EKD. It is known that insulin resistance plays a pivotal role in the development and progression of kidney disease by worsening renal hemodynamics $[3,16,17]$, which can lead to endothelial dysfunction, increased vascular permeability, and sustained glomerular hyperfiltration that ultimately result in abnormal urinary albumin excretion. Insulin resistance may also alter renal cellular metabolism and stimulate mesangial hyperplasia [18]. Moreover, on a background of increased risk for cardiovascular disease as commonly seen in patients with NAFLD, the potential for endothelial dysfunction and renovascular damage may also become more prominent $[19,20]$. In addition, activation of the nuclear factor $(\mathrm{NF})^{-} \kappa \mathrm{B}$ pathway in nonalcoholic steatohepatitis (NASH) has been shown to increase the transcription of a variety of proinflammatory genes that may further amplify systemic chronic inflammation and this mechanism may play a pathogenetic role in the development of CKD [21]. Finally, NASH with varying levels of LF exacerbates systemic and hepatic insulin resistance, causes atherogenic dyslipidemia, and releases a variety of pro-coagulant, pro-oxidant and pro-fibrogenic mediators, which may promote the development and progression of CKD [7, 20, 22, 23]. 
Our study has shown that assessment of LSM is useful to accurately identify patients with NAFLD at risk of EKD. TE has the advantage of providing quantitative measurements of liver stiffness and this reproducible methodology has been validated as a good proxy for LF in different patient populations [24]. Although liver biopsy is considered the "gold standard" for staging LF, this invasive procedure has potential risks of acute complications and is not liked by patients [25]. Consequently, TE is becoming a widely accepted methodology for use in both clinical practice and in research $[24,26,27]$.

For the cut-off used in our study, we showed that the LSM score had appropriate discriminatory power for predicting the histologic stage of LF. In addition, the association between TE-measured LF and risk of EKD was consistent with data obtained from liver biopsy. By combining LSM with age and sex, our novel LSM-EKD model also showed good performance for identifying EKD. This finding is clinically relevant because this LSM-EKD model may be useful to clinicians to screen for risk of CKD in patients with NAFLD.

Our study has some important limitations. Firstly, the cross-sectional design of the study does not allow us to establish temporal and causal relationships between the severity of LF and the presence of EKD. Secondly, we had only a single measurement of both albuminuria and eGFR levels in this study. Thirdly, no information about the specific renal pathology associated with NAFLD was available in our study. In addition, we excluded patients treated with potentially nephro-toxic drugs. However, patients treated with anti-hyperglycaemic or anti-hypertensive drugs that may be potentially nephro-protective were included in the study (but the use of these drugs has been adjusted for in our multivariable logistic regression models). Finally, it is possible that there may be a selection bias because several subjects in our study were excluded due to the stringent exclusion criteria. Thus, we suggest that further research is now needed to test the associations we have observed between the severity of LF and risk of EKD in other ethnic cohorts and to better 
investigate the incidence rates of progression of EKD to macroalbuminuria, impaired eGFR and end-stage renal disease in patients with NAFLD.

In conclusion, the results of our study show that there is a significant positive association between the severity of LF, assessed either by liver biopsy or non-invasively by Fibroscan ${ }^{\circledR}$, and the presence of EKD in patients with non-cirrhotic biopsy-proven NAFLD. This association is independent of common renal risk factors, such as age, sex, ethnicity, measures adiposity, hypertension, dyslipidemia, hyperuricemia, diabetes and HOMA-IR score. In addition, our results also suggest that evaluation of LF, assessed by either histology or by TE, may be useful for accurately identifying NAFLD patients who are at increased risk of EKD. We consider that identifying NAFLD patients at high risk of EKD may assist referral of these patients to tertiary care centers for more careful surveillance (as proposed in Figure 3).

\section{Funding Sources:}

This work was supported by grants from the National Natural Science Foundation of China (81500665), High Level Creative Talents from Department of Public Health in Zhejiang Province, Project of New Century 551 Talent Nurturing in Wenzhou and General Program of Science and Technology Development Foundation of Nanjing Medical University (2017NJMU168). GT is supported in part by grants from the University School of Medicine of Verona, Verona, Italy. CDB is supported in part by the Southampton NIHR Biomedical Research Centre, UK (IS-BRC-20004).

\section{Authors contributions:}

Dan-Qin Sun and Ming-Hua Zheng designed the study, prepared the figures, reviewed the results, interpreted data and wrote the manuscript. Yusuf Yilmaz, Haluk Tarik Kani, Fang-Zhou Ye, Kenneth I. Zheng and Yong-Ping Chen collected data. Jian-Rong Yang contributed reagents and performed the experiments. Hao-Yang Zhang and Wei-Jie Yuan performed statistical analyses. 
Christopher D. Byrne, Giovanni Targher, and Kenneth I. Zheng proofread the manuscript. All authors contributed to the manuscript for important intellectual content and approved the submission.

\section{Disclosure statement:}

All authors have no potential conflicts of interest to disclose.

Part of data had been presented as a poster in 2018 EASL NAFLD Summit, which was held in Geneva, Switzerland, from 20 to 23 September 2018.

\section{Acknowledgments}

We thank Xiao-Yan Pan and Chen-Fei Zheng who contributed by patient referral, thank Pei-Wu Zhu who performed partial experiments, thank Xiao-Dong Wang who analyzed pathological biopsies and also thank Prof. Ji-Min Liu, a liver pathologist from McMaster University, who conducted quality control of pathology data in Wenzhou cohort.

\section{References}

[1] Targher G, Chonchol MB, Byrne CD. CKD and nonalcoholic fatty liver disease. American journal of kidney diseases : the official journal of the National Kidney Foundation 2014;64(4):638-52.

[2] Paik J, Golabi P, Younoszai Z, Mishra A, Trimble G, Younossi ZM. Chronic kidney disease is independently associated with increased mortality in patients with nonalcoholic fatty liver disease. Liver international : official journal of the International Association for the Study of the Liver 2019;39(2):342-52.

[3] Yilmaz Y, Alahdab YO, Yonal O, Kurt R, Kedrah AE, Celikel CA, et al. Microalbuminuria in nondiabetic patients with nonalcoholic fatty liver disease: association with liver fibrosis. Metabolism: clinical and experimental 2010;59(9):1327-30.

[4] Ramspek CL, de Jong Y, Dekker FW, van Diepen M. Towards the best kidney failure prediction tool: a systematic review and selection aid. Nephrology, dialysis, transplantation : official publication of the European Dialysis and Transplant Association - European Renal Association 2019.

[5] Crews DC, Bello AK, Saadi G, for the World Kidney Day Steering C. Burden, Access, and Disparities in Kidney Disease. American journal of nephrology 2019;49(3):254-61.

[6] Coresh J, Heerspink HJL, Sang Y, Matsushita K, Arnlov J, Astor BC, et al. Change in albuminuria and subsequent risk of end-stage kidney disease: an individual participant-level consortium meta-analysis of observational studies. The lancet Diabetes \& endocrinology 2019;7(2):115-27.

[7] Targher G, Byrne CD. Non-alcoholic fatty liver disease: an emerging driving force in chronic kidney disease. Nature reviews Nephrology 2017;13(5):297-310.

[8] Mantovani A, Zaza G, Byrne CD, Lonardo A, Zoppini G, Bonora E, et al. Nonalcoholic fatty liver disease increases risk of incident chronic kidney disease: A systematic review and meta-analysis. Metabolism: clinical and experimental 2018;79:64-76. 
[9] Chinnadurai R, Ritchie J, Green D, Kalra PA. Non-alcoholic fatty liver disease and clinical outcomes in chronic kidney disease. Nephrology, dialysis, transplantation : official publication of the European Dialysis and Transplant Association - European Renal Association 2019;34(3):449-57.

[10] Roulot D, Costes JL, Buyck JF, Warzocha U, Gambier N, Czernichow S, et al. Transient elastography as a screening tool for liver fibrosis and cirrhosis in a community-based population aged over 45 years. Gut 2011;60(7):977-84.

[11] Castera L, Friedrich-Rust M, Loomba R. Noninvasive Assessment of Liver Disease in Patients With Nonalcoholic Fatty Liver Disease. Gastroenterology 2019;156(5):1264-81 e4.

[12] Qin S, Wang S, Wang X, Wang J. Liver stiffness assessed by transient elastography as a potential indicator of chronic kidney disease in patients with nonalcoholic fatty liver disease. Journal of clinical laboratory analysis 2019;33(2):e22657.

[13] Brunt EM, Janney CG, Di Bisceglie AM, Neuschwander-Tetri BA, Bacon BR. Nonalcoholic steatohepatitis: a proposal for grading and staging the histological lesions. The American journal of gastroenterology 1999;94(9):2467-74.

[14] Yeung MW, Wong GL, Choi KC, Luk AO, Kwok R, Shu SS, et al. Advanced liver fibrosis but not steatosis is independently associated with albuminuria in Chinese patients with type 2 diabetes. Journal of hepatology 2017;68(1):147-56.

[15] Levey AS, de Jong PE, Coresh J, El Nahas M, Astor BC, Matsushita K, et al. The definition, classification, and prognosis of chronic kidney disease: a KDIGO Controversies Conference report. Kidney international 2011;80(1):17-28.

[16] Spoto B, Pisano A, Zoccali C. Insulin resistance in chronic kidney disease: a systematic review. Am J Physiol Renal Physiol 2016;311(6):F1087-F108.

[17] Kobayashi H, Tokudome G, Hara Y, Sugano N, Endo S, Suetsugu Y, et al. Insulin resistance is a risk factor for the progression of chronic kidney disease. Clinical nephrology 2009;71(6):643-51.

[18] Parvanova Al, Trevisan R, Iliev IP, Dimitrov BD, Vedovato M, Tiengo A, et al. Insulin resistance and microalbuminuria: a cross-sectional, case-control study of 158 patients with type 2 diabetes and different degrees of urinary albumin excretion. Diabetes 2006;55(5):1456-62.

[19] Kronenberg F. Emerging risk factors and markers of chronic kidney disease progression. Nature reviews Nephrology 2009;5(12):677-89.

[20] Targher G, Lonardo A, Byrne CD. Nonalcoholic fatty liver disease and chronic vascular complications of diabetes mellitus. Nature reviews Endocrinology 2018;14(2):99-114.

[21] Willy JA, Young SK, Stevens JL, Masuoka HC, Wek RC. CHOP links endoplasmic reticulum stress to NF-kappaB activation in the pathogenesis of nonalcoholic steatohepatitis. Mol Biol Cell 2015;26(12):2190-204.

[22] Ix JH, Sharma K. Mechanisms linking obesity, chronic kidney disease, and fatty liver disease: the roles of fetuin-A, adiponectin, and AMPK. Journal of the American Society of Nephrology : JASN 2010;21(3):406-12.

[23] Oberg BP, McMenamin E, Lucas FL, McMonagle E, Morrow J, Ikizler TA, et al. Increased prevalence of oxidant stress and inflammation in patients with moderate to severe chronic kidney disease. Kidney international 2004;65(3):1009-16.

[24] Chow JC, Wong GL, Chan AW, Shu SS, Chan CK, Leung JK, et al. Repeating measurements by transient elastography in non-alcoholic fatty liver disease patients with high liver stiffness. Journal of gastroenterology and hepatology 2019;34(1):241-8.

[25] Araujo AR, Rosso N, Bedogni G, Tiribelli C, Bellentani S. Global epidemiology of non-alcoholic fatty liver disease/non-alcoholic steatohepatitis: What we need in the future. Liver international : official journal of the International Association for the Study of the Liver 2018;38 Suppl 1:47-51.

[26] Dong H, Xu C, Zhou W, Liao Y, Cao J, Li Z, et al. The combination of 5 serum markers compared to FibroScan to predict significant liver fibrosis in patients with chronic hepatitis B virus. Clinica chimica acta; international journal of clinical chemistry 2018;483:145-50.

[27] Siddiqui MS, Vuppalanchi R, Van Natta ML, Hallinan E, Kowdley KV, Abdelmalek M, et al. Vibration-Controlled Transient Elastography to Assess Fibrosis and Steatosis in Patients With Nonalcoholic Fatty Liver Disease. Clinical gastroenterology and hepatology : the official clinical practice journal of the American Gastroenterological Association 2019;17(1):156-63 e2. 


\section{LEGEND TO THE FIGURES}

\section{Figure 1. Study flow diagram}

A total of 742 biopsied participants were initially enrolled, whereas 528 participants who did not meet the inclusion criteria were excluded from the study. Thus, 214 non-cirrhotic patients with biopsy-proven NAFLD were included in the study (154 of these patients also underwent transient elastography).

Figure 2. Area under the ROC curve analyses of the diagnostic performance of LSM score for identifying liver fibrosis on histology (A), as well as the LSM-EKD model (B) and the LFH-EKD model (C) for identifying early kidney dysfunction (EKD) in patients with biopsy-proven NAFLD.

Figure 3. Proposed algorithm for the management of early kidney dysfunction (EKD) in patients with NAFLD. 


\section{TITLES TO THE TABLES}

Table 1. Clinical and biochemical characteristics of patients with biopsy-proven NAFLD, stratified by early kidney dysfunction.

Table 2. Association between early kidney dysfunction and liver fibrosis assessed by either biopsy or transient elastography (Fibroscan $\left.{ }^{\circledR}\right)$.

Table 3. Clinical and biochemical characteristics of patients with NAFLD, stratified by liver fibrosis assessed by transient elastography.

Supplementary table 1. Clinical and biochemical characteristics of patients with NAFLD, stratified by liver fibrosis assessed by liver biopsy. 